\title{
Focus on memory
}

\author{
Nature Neuroscience presents a special focus issue highlighting recent advances and discussing future directions \\ in memory research.
}

W hether remote or recent, blurred or vivid, conscious or hidden, memories provide a link between the present and the past and allow us to project our thoughts into the future. But memories are not immutable; instead, they continually evolve throughout their lifetime. From the moment they are created, they embark on a dynamic journey, during which they are consolidated, often updated, but also sometimes distorted to the point that they falsify the past. As our brain is constantly bombarded with newer information, memories may also become suppressed by competing memories or experiences or seemingly disappear into oblivion. In this issue, we present a focus on memory, comprising Commentaries, Reviews and Perspectives discussing some of the most exciting recent developments and emerging ideas in our understanding of the neurobiology of learning and memory.

Are memories faithful snapshots of past experiences or can they become insidiously deceitful? During its ruling on the Henderson case in 2011, the New Jersey Supreme Court carefully considered the value of evidence based on eyewitness memory. The decision showed some appreciation of the complexity of memory processes and, in particular, the fact that memories can be tampered with by suggestive influence. As a result of this case, instructions provided to jurors on how to decide the fate of the accused in New Jersey now explicitly state that memories do not replay like video recordings. In their Commentary on page 119 of this issue, Daniel Schacter and Elizabeth Loftus carefully discuss what cognitive neuroscience can bring to the courtroom, focusing on the neural basis of true and false memories and misinformation effects. Their commentary also serves as a powerful case to illustrate how advances in understanding the neurobiology of memory have immediate and direct consequences on society.

How are memories transformed by experience? Past experiences can persistently modify gene expression by altering epigenetic marks on histones or DNA bases. The possibility that this form of molecular memory could contribute to the encoding, updating and persistence of long-term memories is very appealing, as memory formation depends on changes in patterns of gene expression. This has inspired memory researchers to investigate whether such mechanisms can participate in shaping learning and memory. In a Perspective on page 124, Matthew Lattal and Marcello Wood discuss recent progress on the epigenetics of learning and memory. They argue that epigenetics can help to distinguish the molecular mechanisms that mediate reconsolidation of memories and memory extinction, and that epigenetics can provide a plausible molecular mechanism that maintains the persistent change in behavior induced by extinction.

Of the many brain structures that are important for learning and memory, the hippocampus has been widely studied as a central hub of this cognitive process. The discovery in the early 1970s by O'Keefe and Dostrovsky that the position of an animal in its environment can dictate the firing of hippocampal neurons, the so-called place cells, prompted the burning question of whether memory and navigation are part of a common system or simply functionally stand side by side in the hippocampus. On page 130 of this issue, György Buzsáki and Edvard Moser revisit this notion by discussing recent evidence that the navigation and memory functions of the hippocampus and the entorhinal cortex are supported by the same neuronal algorithms. In addition, they propose that the mechanisms fueling the memory and mental travel engines of the hippocampal-entorhinal system evolved from the mechanisms supporting navigation in the physical world.

There is now converging and unequivocal experimental evidence supporting the idea that memory consolidation takes place during sleep. Does sleep provide passive protection from forgetting or does it actively shape the future of memories? On page 139 of this issue, Robert Stickgold and Matthew Walker offer a fresh perspective on this question by introducing the concept of sleep-dependent memory triage. They discuss recent findings in sleep research that support their view that consolidation is not a monolithic process, but rather consists of a variety of operations. They propose that offline consolidation during sleep sanctions what information is ultimately retained or lost on the basis of salience tags and acts as the architect of the evolution of memories during their integration into existing implicit and explicit knowledge.

With a long and rich experimental history, learned fear is probably one of the best understood forms of memory. In recent years, it has become clear that aversive memories are shaped by distinct learning phases, and many of the underlying molecular and circuit mechanisms have now been elucidated. On page 146 of this issue, Ryan Parsons and Kerry Ressler review how the latest insights into the biological basis of learned fear have provided a new strategic platform for clinical approaches to fear and anxiety disorders. In particular, they discuss the potential therapeutic value of targeting behavioral and pharmacological interventions to different epochs of the fear learning process to treat disorders such as phobias, panic and post-traumatic stress disorder.

Learning and memory is a very broad field. The handful of pieces in this issue clearly cannot span the entire field; rather, we have simply highlighted some of the topics that have received substantial attention and that have been the focus of recent study. We express our gratitude to the authors, referees and advisors who helped us to curate this special issue. We hope that these reviews will inspire further research into unraveling the complex neuroscience of memory and that they give our readers a glimpse of some of the exciting recent research in this field. 